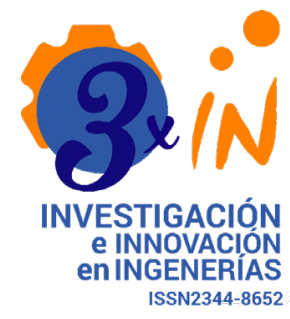

Recibido: 22/09/2020

Aceptado: 22/10/2020

Publicado: 23/11/2020

Correspondencia de autores: gchanchig@unicartagena.edu.co

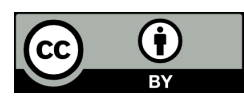

Copyrith 2020

by Investigación e

Innovación en Ingenierías

\section{Arquitectura loT para el desarrollo de sistemas de monitorización y análisis de variables fisiológicas en el área de asistencia médica}

\section{IOT architecture for the development of monitoring and analysis systems of physiological variables in the medical assistance area}

\author{
Gabriel Elías Chanchí Golondrino (iD Manuel Alejandro Ospina Alarcón \\ Martín Emilio Monroy Ríos \\ Universidad de Cartagena, Colombia ID
}

Resumen

Objetivo: Proponer una arquitectura IoT para la obtención, seguimiento y ap rovechamiento de los datos pertenecientes a variables fisiológicas de un paciente (ritmo cordíaco y nivel de oxígeno) mediante dispositivos wearables comerciales para el servicio asistencial en salud. Metodología : Tomando como referencia la arquitectura convencional de IOT a cuatro capas (captura, almacenamiento, análisis y visualización) se diseñó una arquitectura a tres vistas (negocio, funcional e implementación) para el desarrollo de servicios de monitorización de variables fisiológicas que aprovecha los datos de los dispositivos comerciales para la obtención de información de valor agregado dirigida a personal especializado. Resultados: Como resultado de la arquitectura propuesta, se desarrolló una instancia de la misma a través de un sistema IoT para la monitorización y análisis del nivel de oxígeno en la sangre y del ritmo cardíaco. Conclusiones: Los resultados obtenidos demostraron que la arquitectura sirve de referencia para la construcción de sistemas de monitoreo y análisis de variables fisiológicas en el área de la asistencia médica.

Palabras clave: Arquitectura iot, asistencia en salud, monitorización, wearable, ritmo cardíaco, nivel de oxígeno

Abstract

Objective: Propose an IoT architecture for obtaining, monitoring and taking advantage of data pertaining to physiological variables of a patient (heart rhythm and oxygen level) through commercial wearable devices for health care service. Methodology: Taking as a reference the conventional fourlayer IoT architecture (capture, storage, analysis and visualization), a three-view architecture (business, functional and implementation) was designed for the development of physiological variables monitoring services that take advantage of the data from the commercial devices for obtaining valueadded information aimed at specialized personnel. Results: As a result, a reference architecture was obtained, which was validated by developing an IOT system for monitoring and analyzing of both the blood oxygen level and heart rate. Conclusions: The results obtained demonstrate that the proposed architecture serves as a reference for the construction of monitoring and analysis systems of physiological variables in the health assistance service.

Keywords: IOt architecture, health assistance, monitoring, wearable, heart rate, oxygen level.

Como citar (IEEE): G. Chanchí-Golondrino., M. Ospina-Alarcón., y M. Monroy Ríos. “Arquitectura IoT para el desarrollo de sistemas de monitorización y análisis de variables fisiológicas en el área de asistencia médica". Investigación e Innovación en Ingenierías, vol. 8, n³, 1-13, 2020. DOI: https://doi.org/10.17081/invinno.8.3.4699 


\section{Introducción}

Internet de las cosas (IOT) puede definirse como la conexión de objetos físicos o dispositivos en una red abierta que tiene la capacidad de auto gestionarse, compartir información, datos y recursos, reaccionar y actuar frente a situaciones y cambios en el entorno [1, 2]. A partir de la gran difusión de loT en diferentes contextos de aplicación, uno de los desafíos más importantes es el aprovechamiento de los grandes volúmenes de datos de cara a la obtención de información útil para la toma de decisiones [3].

En la implementación de los sistemas IOT para diversos contextos de aplicación, se suele hacer uso de arquitecturas compuestas por 4 capas funcionales: captura, almacenamiento, análisis y visualización de datos $[4,5,6]$. La capa de captura está compuesta por sensores o dispositivos encargados de la obtención de los datos asociados a diferentes tipos de variables, dependiendo del contexto de aplicación. La capa de almacenamiento, por su parte, está conformada por tecnologías que permiten guardar los datos capturados en tiempo real. En la capa de análisis se hace uso de diferentes técnicas y tecnologías, que permiten la aplicación de modelos de analítica de datos (arboles de decisión, reglas de inferencia, clustering, etc.). Finalmente, en la capa de visualización se hace uso de tecnologías que permiten presentar de manera gráfica la información histórica y en tiempo real capturada [7, 8]. En este sentido, las diferentes capas de la arquitectura pueden ser particularizadas dependiendo del contexto de aplicación.

Uno de los campos de mayor potencialidad de aplicación de loT es el servicio asistencial en salud [9], para el cual se han desarrollado diferentes tipos de dispositivos por parte de diversos fabricantes, con el objetivo de obtener datos de variables fisiológicas de los pacientes en tiempo real y transmitirlos usando diferentes protocolos de comunicación ampliamente difundidos, como es el caso de bluetooth LE (BLE) [10]. A pesar de lo anterior, dichos dispositivos tienen los siguientes problemas: 1) dificultad para almacenar información de manera constante, 2) están asociados a aplicaciones móviles cuyos datos no son abiertos, 3) se dificulta la aplicación de modelos de analítica de datos y 4) es difícil obtener información enriquecida que puede ser usada por los médicos o personal especializado, de tal manera que se aproveche completamente las potencialidades de IoT.

Del mismo modo han surgido diferentes alternativas desde el campo del hardware libre, las cuales a pesar de que posibilitan la implementación de sistemas de seguimiento, tienen aún como desafío la portabilidad [11]. Por otra parte, el monitoreo es considerado uno de los aspectos más importantes en los sistemas de salud soportados en IoT [12]. Asimismo, partiendo de los dispositivos comerciales existentes, se hace necesaria la propuesta de una arquitectura IoT para el desarrollo de sistemas de monitorización y análisis en el contexto de la salud, la cual permita guiar el proceso de desarrollo, especificando las diferentes técnicas y tecnologías que pueden emplearse en las diferentes vistas de la arquitectura convencional de IOT $[4,5,6]$. Además, la revisión que se hizo de la literatura, cuyos resultados se exponen en la sección de trabajos relacionados, conduce a la necesidad de definir una arquitectura que sirva de referencia para el desarrollo de sistemas de monitorización y análisis de variables fisiológicas en el área de la asistencia médica.

En este orden de ideas, se propone como contribución una arquitectura para el desarrollo de sistemas de monitorización loT en el contexto del servicio asistencial en salud, la cual sirve de referencia para la creación de servicios y aplicaciones en esta área, aprovechando las ventajas de los dispositivos loT comerciales y generando información de valor a partir de los datos capturados. Esta arquitectura fue definida mediante tres vistas: vista de negocio, vista funcional y vista de implementación. Cada vista contempla las cuatro capas de la arquitectura convencional de IoT [4]. Como técnica de validación de la arquitectura se utilizó la 
Arquitectura IoT para el desarrollo de sistemas de monitorización y análisis de variables fisiológicas en el área de asistencia médica

creación de un prototipo [13] para la monitorización del ritmo cordíaco y del nivel de oxígeno en la sangre, el cual además de posibilitar el seguimiento de dichas variables, permite almacenar las sesiones de captura y analizarlas mediante modelos de clustering

En adelante el artículo está organizado de la siguiente forma: inicialmente se presenta la metodología usada para el desarrollo de la investigación; seguidamente se describen un conjunto de trabajos relacionados que se tuvieron en cuenta para la definición de la arquitectura; posteriormente se explica la arquitectura loT para el desarrollo de sistemas de monitorización en salud, como resultado principal de la investigación; a continuación se presenta la validación de la arquitectura por medio del desarrollo de un prototipo para la monitorización y el análisis del ritmo cardíaco y el nivel de oxígeno en la sangre, haciéndose la discusión de los resultados; finalmente se muestran las conclusiones y trabajos futuros derivados de la investigación.

\section{Metodología}

En la Figura 1 se presentan las cuatro fases metodológicas que guiaron el proceso de definición de las diferentes vistas arquitectónicas de la arquitectura y la construcción del prototipo derivado de la misma [14]. En la fase 1 de la metodología se definieron los elementos principales de la vista de negocio, de tal modo que se ilustra el proceso de funcionamiento de los sistemas de monitorización lot en salud, desde el punto de vista de los agentes que intervienen. En la fase 2 se definen los módulos funcionales asociados a las diferentes capas de la arquitectura convencional de IoT [4]. En la fase 3 se exploraron un conjunto de técnicas y tecnologías adecuadas para la implementación de los módulos funcionales definidos en la fase 2. Finalmente, en la fase 4, se realizó la validación de la arquitectura propuesta mediante creación de un prototipo [13] de sistema loT para el seguimiento del nivel de oxígeno en la sangre y el ritmo cordíaco.

Figura 1. Metodología considerada

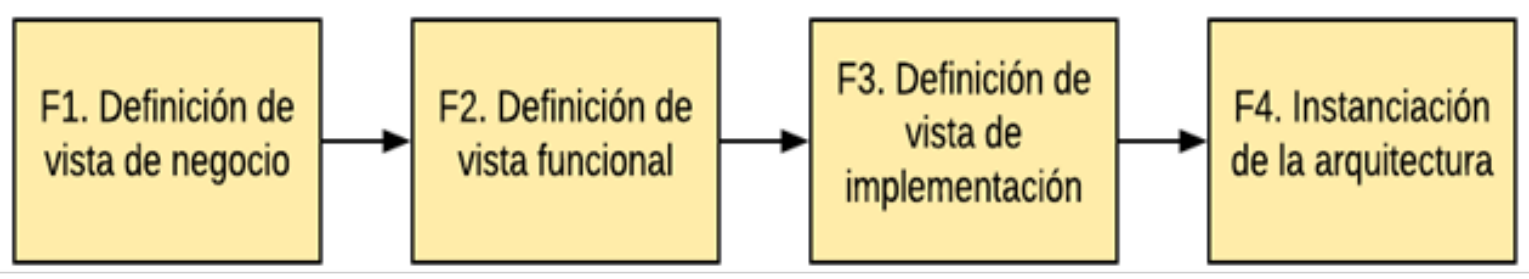

Fuente: Elaboración propia

\section{Trabajos Relacionados}

Delgado et al. presentan un sistema hardware-software para la captura del ritmo cordíaco y su clasificación en tres categorías bradicardia, ritmo normal y taquicardia [11]. Así mismo, a partir de los datos obtenidos en un periodo de tiempo, el sistema permite determinar el estrés mental partiendo de la variabilidad del ritmo cardíaco. El sistema propuesto fue construido haciendo uso de una placa del hardware libre Arduino Mega, un sensor de ritmo cardíaco y una pantalla LCD. Aunque la principal ventaja de este sistema es la portabilidad, no permite el almacenamiento de los datos de las sesiones de captura realizadas, ni tampoco el análisis de los datos capturados mediante modelos de analítica. 
Chanchí, Giraldo y Campo proponen un sistema software para la monitorización del estrés mental de un usuario a lo largo de una prueba de usabilidad [15]. El sistema propuesto por los autores, obtiene a lo largo de la prueba las variables fisiológicas de ritmo cordíaco y variabilidad del ritmo cordíaco mediante un cinturón bluetooth. A partir de los datos capturados, el sistema realiza el cálculo del estrés mental de un usuario cada 5 segundos y a lo largo de la prueba. Este sistema no guarda la información de las diferentes sesiones, ni posibilita la aplicación de modelos de analítica de datos con las capturas realizadas.

Gutierrez-Ardila et al. desarrollan un sistema IoT basado en arquitecturas orientadas a servicios [16], el cual permite al paciente realizar chequeos preventivos haciendo uso de un dispositivo Android y una banda de ritmo cardíaco. La solución propuesta hace uso de una metodología de análisis basada en teoría de la probabilidad, a través de la cual es posible generar un diagnóstico preventivo sobre las afecciones cardíacas. El sistema propuesto tiene como aporte la identificación de los principales módulos funcionales de un sistema de seguimiento loT en el campo de la salud. Del mismo modo, el sistema hace uso de bases de datos convencionales y no considera el uso de modelos de analítica de datos.

Farahani et al. proponen una arquitectura holística para ecosistemas de salud basada en IoT [17], en la que se cambia el paradigma de la atención médica centrada en la clínica, por un nuevo enfoque donde la atención se centra en el paciente, en el que surgen nuevos retos como: la gestión de datos, la escalabilidad, las regulaciones, la interoperabilidad, las interfaces humano-dispositivo-red, la seguridad y la privacidad. Este ecosistema de IoT eHealth requiere de una arquitectura de múltiples capas: 1) dispositivo, 2) computación en la niebla y 3) computación en la nube para potenciar el manejo de datos complejos en términos de su variedad, velocidad y latencia. Esta solución aborda el problema desde un alto nivel de abstracción sin revelar detalles relacionados con el monitoreo y el análisis de las variables fisiológicas capturadas.

Kumar propone una arquitectura para sistemas de salud de extremo a extremo [18], soportados en IoT usando el módulo Intel Curie. Esta solución centra más la atención en el hardware. Del mismo modo, en [10] los autores realizan una revisión sobre los diferentes conceptos, tecnologías, arquitecturas convencionales y escenarios típicos de aplicación de los sistemas loT en el contexto de la salud. Los autores concluyen que ante la gran cantidad de tecnologías y dispositivos soportados en IOT y aportados por diferentes fabricantes, se hace necesario aprovechar el desarrollo de servicios y promover la generación de arquitecturas integradoras en el campo de la salud. Dentro de las arquitecturas convencionales exploradas por los autores, no se evidencia el uso de la arquitectura por capas, por lo que se cumplen de manera parcial o incompleta las funcionalidades de cada una de las capas consideradas en el presente trabajo.

En [19] se propone una plataforma IoT para enfermedades no transmisibles denominada eBPlatform. Esta plataforma se desarrolló en China, para brindar atención oportuna a la gran cantidad de pacientes con este tipo de enfermedades, considerando que baja capacidad del sistema de salud dificulta su atención. La plataforma fue diseñada para obtener desde el hogar del paciente variables fisiológicas tales como: presión en la sangre, nivel de azúcar en la sangre y ritmo cordíaco, las cuales son enviadas a un servidor remoto donde son almacenados y puestos a disposición de un médico para su posterior análisis. La arquitectura de la plataforma propuesta está organizada en tres capas: i) sensores, ii) procesamiento de datos y iii) aplicaciones, de tal modo que esta arquitrectura y/o plataforma tiene como limitante la inclusión de las funcionalidades asociadas a los modelos para el análisis de datos.

En consecuencia, se observa la necesidad de definir una arquitectura que sirva de referencia para el desarrollo de sistemas de monitorización y análisis de variables fisiológicas en el área de la asistencia médica. 
Arquitectura IoT para el desarrollo de sistemas de monitorización y análisis de variables fisiológicas en el área de asistencia médica

\section{Arquitectura del Sistema loT}

En esta sección se presenta la descripción de la arquitectura propuesta para la construcción de sistemas de monitorización y análisis dentro del servicio asistencial en salud. La arquitectura fue organizada en tres vistas: i) vista de negocio, ii) vista funcional y iii) vista de implementación, cada una de las cuales está conformada por las cuatro capas de la arquitectura convencional de IoT [4, 5, 6]. La vista de negocio muestra el proceso de interacción de los diferentes agentes involucrados con el sistema loT. En la vista funcional se muestran los diferentes módulos funcionales asociados a las cuatro capas convencionales de la arquitectura IoT. Finalmente, en la capa de implementación se presentan un conjunto de tecnologías que permiten el cumplimiento de las funcionalidades especificadas en la vista funcional.

\section{Vista de negocio}

En la Figura 2 se presenta el diagrama que ilustra la vista de negocio de la arquitectura para sistemas IoT de monitorización en el servicio asistencial en salud. Tal como se aprecia en la Figura 2, los dispositivos, tecnologías y técnicas empleadas en la vista de negocio se enmarcan en las cuatro capas convencionales de la arquitectura IoT: captura, almacenamiento, visualización y análisis.

Figura2. Vista de negocio

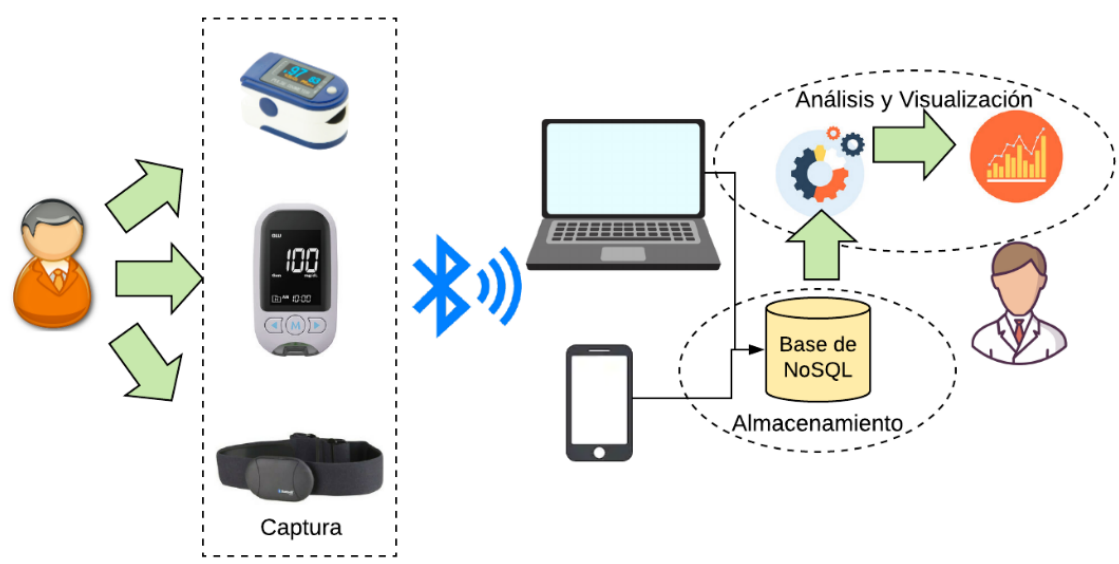

Fuente: Elaboración propia

Dentro de la vista de negocio de la arquitectura propuesta, el usuario final tiene articulado a su espacio corporal un conjunto de dispositivos wearables que permiten la obtención en tiempo real de diferentes variables fisiológicas tales como: ritmo cardíaco, variabilidad del ritmo cardíaco, nivel de oxígeno en la sangre, nivel de azúcar en la sangre, etc. Estas variables son transmitidas en tiempo real por los dispositivos wearables haciendo uso del protocolo de comunicación inalámbrica de bajo consumo de energía bluetooth LE, hacia equipos receptores con soporte para este protocolo (dispositivos móviles, computadores con receptor bluetooth, etc). Para la obtención de los datos asociados a las variables fisiológicas, los equipos receptores además de contar con el soporte hardware para la recepción (adaptadores, hardware incluido) hacen uso de librerías que posibilitan la obtención de la trama de datos, que son decodificados (dependiendo los servicios y características ofrecidas por cada dispositivo) y almacenados en una base de datos NoSQL, la cual hace posible la escalabilidad y posterior visualización de los datos. A partir de los datos almacenados, se aplican modelos de analítica de datos que permiten obtener información útil de las diferentes sesiones de captura realizadas. Esta información puede contribuir a mejorar el diagnóstico de diferentes tipos de afecciones, siendo las afecciones cardíacas el caso de mejor correspondencia con los dispositivos comerciales más comunes y sus variables asociadas. 


\section{Vista Funcional}

En la Figura 3 se presenta el diagrama de flujo de la vista funcional perteneciente a la arquitectura del sistema loT para la monitorización del servicio asistencial en salud. En este diagrama de flujo, cada una de las capas de la vista funcional incluyen un conjunto de bloques que representan las operaciones de: ejecución de los procesos de captura, seguimiento, almacenamiento, visualización y análisis.

Figura 3. Vista funcional

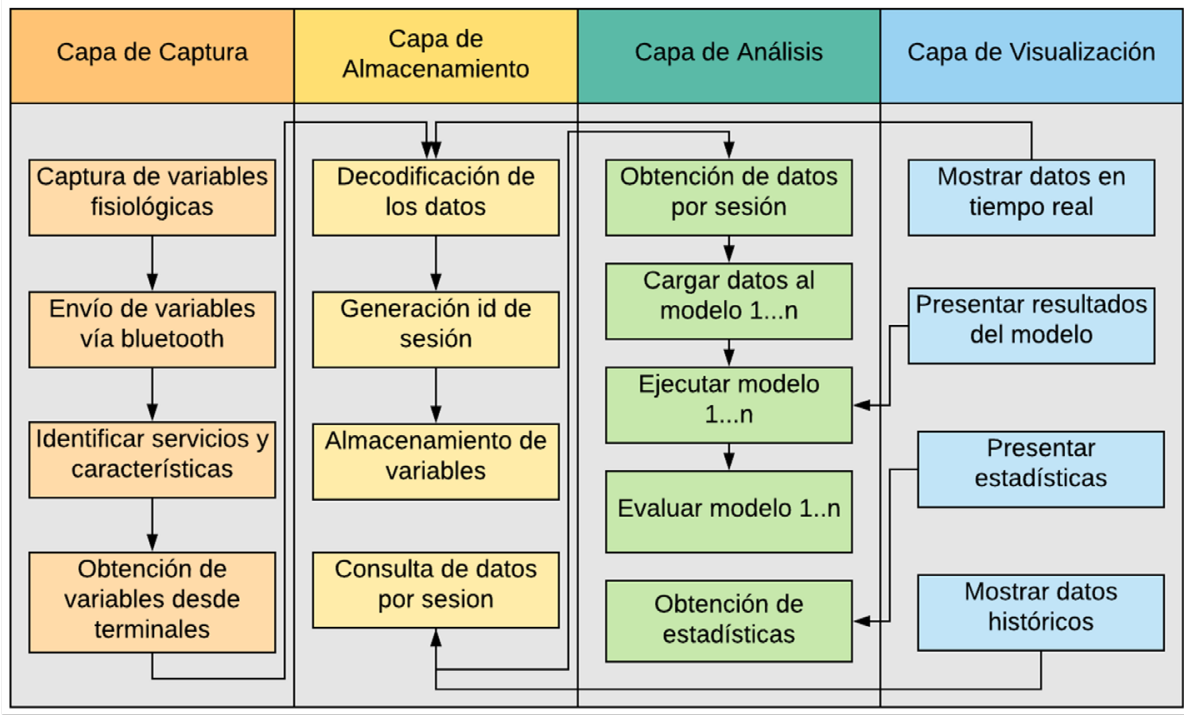

\section{Fuente: Elaboración propia}

En la capa de captura en primera instancia se obtienen un conjunto de variables fisiológicas desde los dispositivos wearables comerciales, las cuales una vez obtenidas son enviadas a través del protocolo de comunicación inalámbrica bluetooth LE a los equipos receptores. Desde los terminales o equipos receptores (dispositivos móviles, computador), es necesario identificar los id o códigos de acceso a los servicios y características bluetooth LE disponibles en cada uno de los dispositivos, con el fin de obtener las variables fisiológicas transmitidas de manera inalámbrica. En la capa de almacenamiento una vez los datos son obtenidos por los terminales o equipos receptores, son decodificados a partir de la trama de datos capturada en formato hexadecimal. Cada conjunto de datos capturados por paciente desde los dispositivos wereables es asociado a una sesión de captura, por lo cual los datos decodificados son almacenados teniendo un id de la sesión en una base de datos no relacional. De igual modo, al mismo tiempo que la capa de almacenamiento permite guardar las variables obtenidas en una sesión de usuario, también posibilita la consulta de los datos almacenados por parte de las capas de análisis y visualización. En la capa de análisis se realizan cálculos estadísticos básicos a partir del historial de datos capturados y se aplican modelos de análisis de datos, para lo cual se cargan los datos al modelo, se ejecuta el modelo y se evalúa la precisión del mismo, haciendo uso de librería de minería de datos. Finalmente, en la capa de visualización, es posible realizar un seguimiento gráfico de las variables fisiológicas obtenidas en la capa de captura. Del mismo modo en esta vista se presentan los resultados de las estadísticas halladas, así como los resultados de la ejecución y evaluación de los modelos de análisis considerados. 
Arquitectura IoT para el desarrollo de sistemas de monitorización y análisis de variables fisiológicas en el área de asistencia médica

\section{Vista de Implementación}

En la Figura 4 por su parte, se presenta la vista de implementación de la arquitectura loT para el desarrollo de sistemas de monitorización. Esta vista presenta un conjunto de técnicas y tecnologías asociadas a cada capa, las cuales buscan dar cumplimiento a las funcionalidades presentadas en la Figura 3.

Figura 4. Vista de implementación

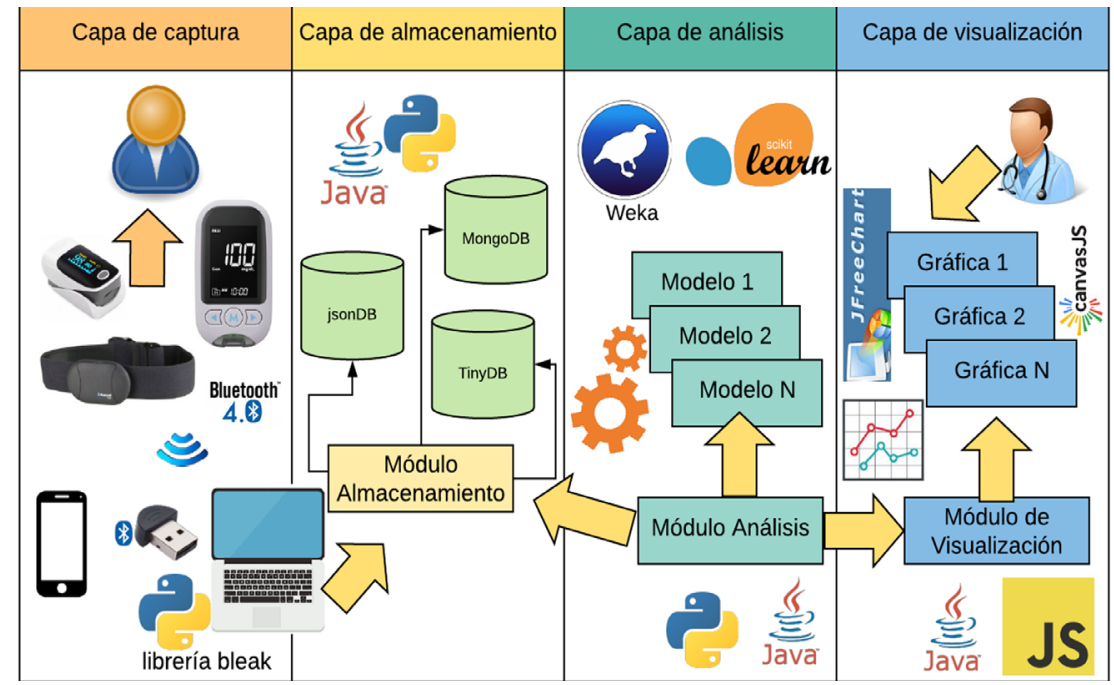

Fuente: Elaboración propia

En la capa de captura mediante un conjunto de dispositivos comerciales tipo wearable, se obtienen diferentes variables fisiológicas de un paciente, las cuales son enviadas a equipos receptores mediante bluetooth LE. Para realizar la conexión a estos dispositivos se hace uso de la librería bleak de Python, la cual permite que desde el sistema operativo Windows y Linux se pueda realizar la conexión asíncrona a los dispositivos wearables. Una vez los datos son obtenidos y decodificados desde los equipos receptores, pueden ser guardados en bases de datos no relacionales mediante el módulo de almacenamiento usando un id de sesión de captura. Dentro de estas bases de datos se consideran: jsonDB, MongoDB, TinyDB, etc. A partir de los datos almacenados en la base de datos, en la capa de análisis el módulo de análisis se encarga de cargar los datos y ejecutar diferentes algoritmos de análisis. Dependiendo del tipo de lenguaje de programación, se pueden usar diferentes alternativas para el análisis de datos como puede ser la librería weka de Java, la librería scikit learn de Python entre otras. Finalmente, en la capa de visualización mediante el módulo de visualización se presentan las variables en tiempo real, así como los datos históricos y los resultados de la aplicación de los modelos de análisis. Estos resultados pueden ser de interés para médicos y especialistas del servicio asistencial en salud. 


\section{Resultados}

\section{Validación de la arquitectura loT}

Como técnica de validación de la arquitectura propuesta se utilizó la construcción de prototipos [13], y para el prototipo desarrollado se diseñaron y aplicaron casos de prueba para los escenarios correspondientes a las funcionalidades básicas de captura, seguimiento y análisis del nivel de oxígeno en la sangre. En la Figura 5 se detalla un diagrama que representa la vista específica de implementación del prototipo desarrollado.

Figura 5. Vista de implementación del prototipo

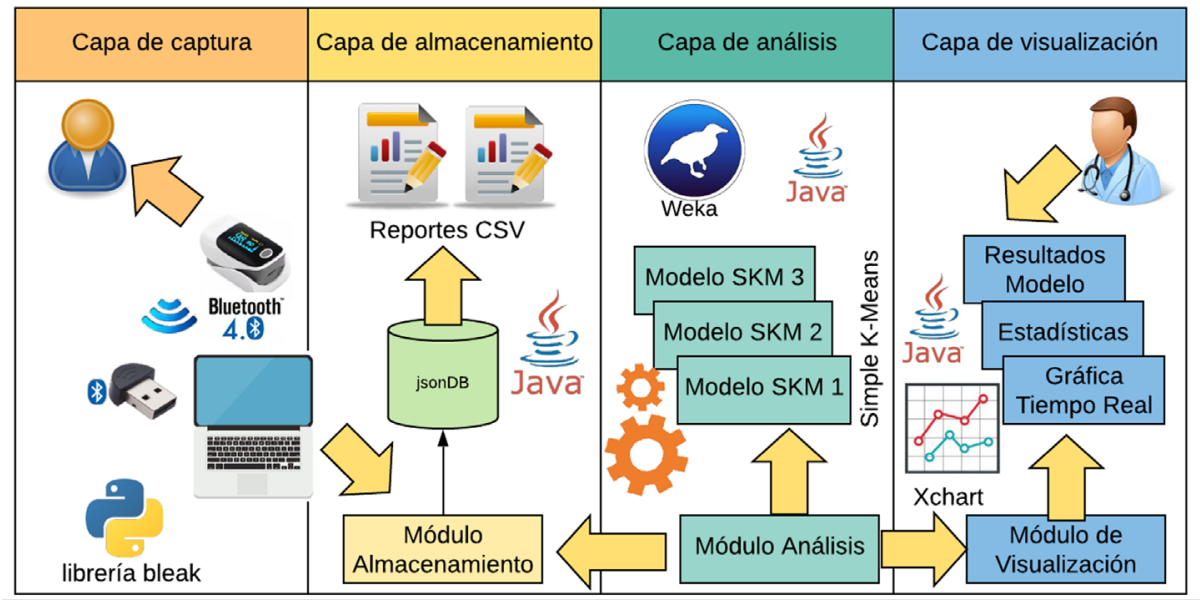

Fuente: Elaboración propia

En la capa de captura se cuenta con un oxímetro IoT comercial que se coloca en el dedo del usuario y permite obtener las variables fisiológicas de: ritmo cardíaco, el nivel de oxígeno en la sangre (SpO2) y el índice de perfusión (PI). De igual modo a partir del ritmo cardíaco es posible obtener la variabilidad del ritmo cardíaco o intervalo RR, dividiendo 60 entre el valor del ritmo cordíaco. Estas variables son enviadas de manera asincrónica por el oxímetro a los dispositivos receptores usando bluetooth LE. En el dispositivo receptor por su parte, se cuenta con un adaptador USB bluetooth para la recepción y un script de Python que haciendo uso de la librería bleak de Python permite acceder a los servicios y características provistas por el dispositivo.

Esta librería tiene como ventaja el hecho de que permite el acceso a los servicios de dispositivos comerciales tanto desde el sistema operativo Windows como de Linux. El script de Python es invocado en segundo plano mientras dura la sesión mediante un aplicativo desarrollado en Java, el cual se encarga de la gestión del almacenamiento, análisis y visualización de los datos. De este modo, desde el aplicativo Java y a través de un módulo de almacenamiento, los datos capturados se almacenan en la base de datos NoSQL jsonDB. Cada grupo de datos es asociado con un id de la sesión de captura y una estampa de tiempo del momento en el que fueron capturados los datos (datos asincrónicos). En lo referente a la capa de análisis, el aplicativo Java carga los datos de las sesiones a diferentes modelos basados en el algoritmo Simple K-Means, los cuales fueron construidos haciendo uso de la librería de minería de datos weka. Los modelos de análisis se encargan a partir de los datos de una sesión de captura de relacionar las diferentes variables fisiológicas entre si y con la estampa de tiempo obtenida, con el fin de identificar los centroides de los datos capturados. Finalmente, en la capa de visualización, el aplicativo Java presenta en pantalla los datos capturados en tiempo real haciendo uso de la librería XChart de Java, así como los resultados obtenidos mediante los modelos de análisis y los resultados estadísticos de las variables fisiológicas de cada sesión (promedio, mayor, menor, etc.). 
Arquitectura IoT para el desarrollo de sistemas de monitorización y análisis de variables fisiológicas en el área de asistencia médica

A partir de la vista de implementación presentada en la Figura 5, en la Figura 6 se muestra la interfaz principal del sistema de monitorización en funcionamiento, desarrollado en el lenguaje de programación Java.

Figura 6. Sistema loT de monitorización de oxígeno en la sangre.

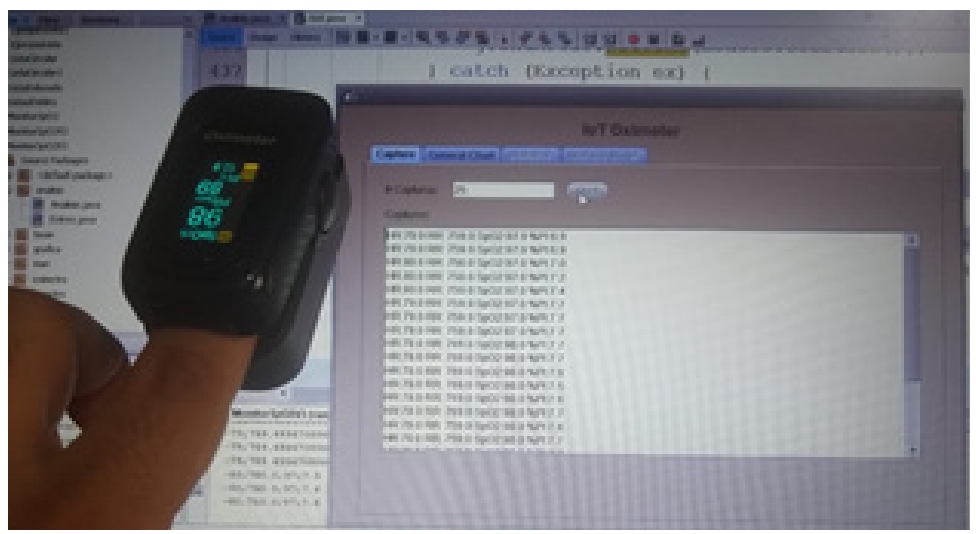

\section{Fuente: Elaboración propia}

Tal como se aprecia en la Figura 6, el sistema implementado a partir de la arquitectura consta de las siguientes cuatro pestañas: "Capture", "General Chart", "Statistics" y "Analysis Model". En la pestaña "Capture" se configuran el número de capturas asincrónicas a realizar a partir del oxímetro, de tal modo que al presionar el botón "Start" se inicia la captura de la trama de datos desde el dispositivo bluetooth, haciendo uso en segundo plano de un script en Python que posibilita la conexión al oxímetro mediante la librería bleak. Para el caso del oxímetro de la Figura 6, el script de Python se conecta a la dirección física del dispositivo y recibe las notificaciones de la característica 0000fee1-0000-1000-8000-00805f9b34fb. Al tiempo que la trama de datos es recibida y decodificada, se presentan en el área de texto de la pestaña "Capture" los datos de: ritmo cardíaco (HR), variabilidad del ritmo cardíaco (RR), nivel de oxígeno (SpO2) e índice de perfusión (PI). De manera simultánea a que los datos se presentan en pantalla, son almacenados con un id de sesión generado al presionar el botón "Start" y una estampa de tiempo relacionada con el momento en el que se van obteniendo las variables fisiológicas. Así, en la Figura 7 se muestran los registros almacenados en la base de datos jsonDB, donde cada registro tiene asociado un id de sesión.

Figura 7. Registro de datos en la base de datos jsondb

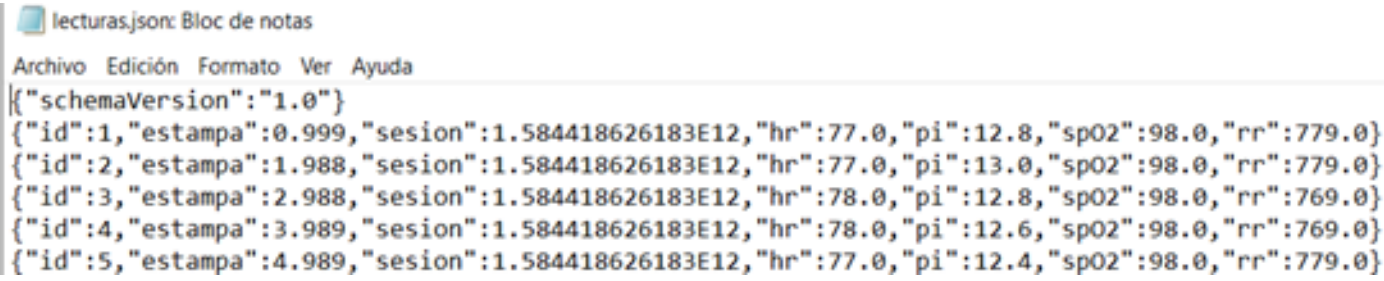

\section{Fuente: Elaboración propia}


Figura 8. Seguimiento en tiempo real del ritmo cardíaco

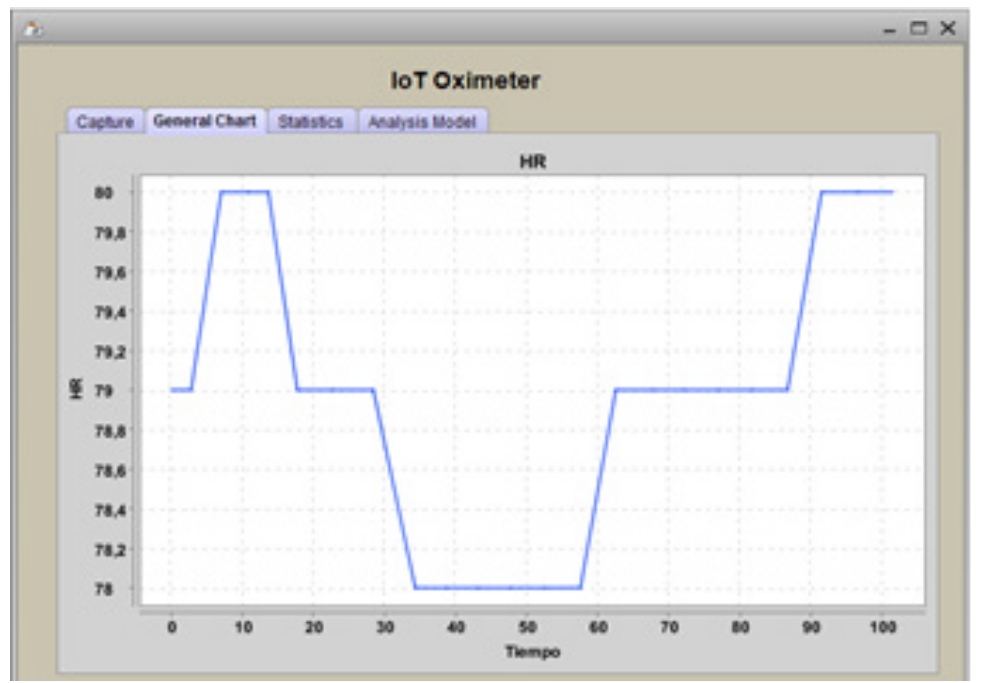

\section{Fuente: Elaboración propia}

Del mismo modo, en la pestaña "General Chart", a medida que se van obteniendo los datos se va presentado el comportamiento del ritmo cordíaco en el tiempo, hasta que se obtienen el número de capturas especificadas en la pestaña "Capture" (ver Figura 8). La gráfica en tiempo real generada en esta pestaña fue desarrollada haciendo uso de la librería XChart de Java.

Una vez se han terminado de recibir el número de capturas establecidas en la pestaña "Capture", en la pestaña "Statistics" es posible realizar una consulta sobre las diferentes sesiones capturadas con el fin de acceder a los datos de las variables fisiológicas obtenidas en el tiempo (estampa de tiempo obtenida) (ver Figura 9).

\section{Figura 9. Estadísticas sobre las sesiones de captura}

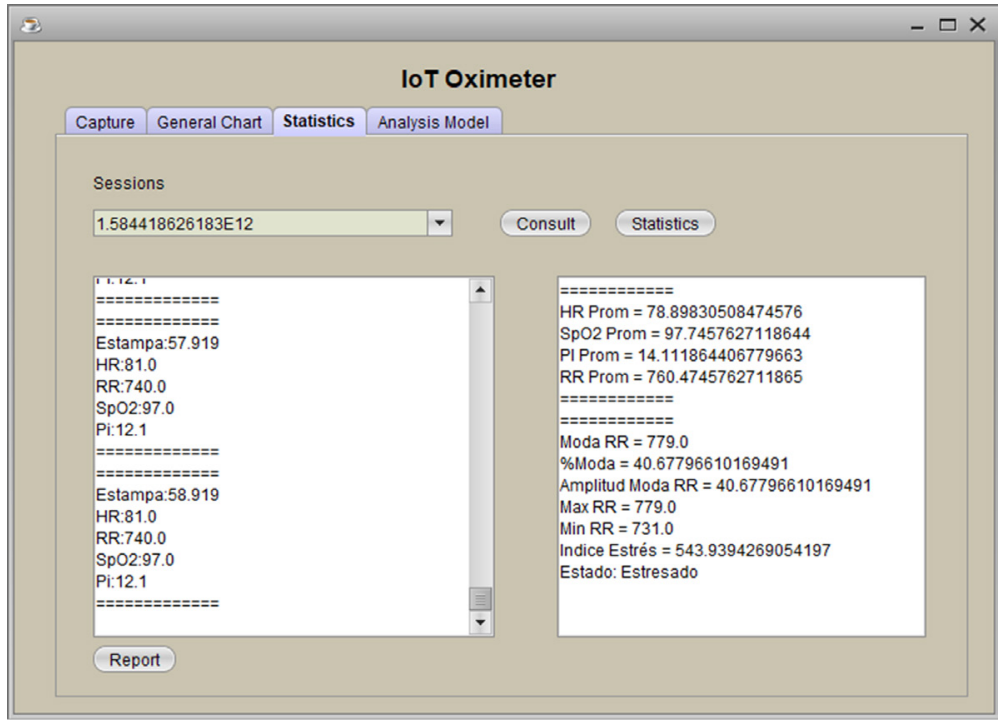

Fuente: Elaboración propia 
Arquitectura IoT para el desarrollo de sistemas de monitorización y análisis de variables fisiológicas en el área de asistencia médica

De este modo, sobre los datos de cada sesión es posible obtener el promedio de cada una de las variables fisiológicas obtenidos durante dicha sesión. Adicionalmente, a partir de los datos calculados de los intervalos RR (obtenidos mediante el ritmo cardíaco) se calcula el índice de estrés mental usando la ecuación de Bayevsky presentada en [20]. De este modo, la sesión de ejemplo presentada en la Figura 9 tiene una duración de 58 segundos, en la cual el promedio del ritmo cardíaco es de 78.898, el promedio del nivel de oxígeno es de 97.7457 , el promedio del índice de perfusión es de 14.11 y el promedio de los intervalos RR es de 760.474. Del mismo modo el índice de estrés mental calculado es de 543.94 lo que corresponde al nivel estresado. Finalmente, en la pestaña de "Analysis Model" es posible aplicar diferentes modelos de análisis basados en clustering a las sesiones de captura almacenadas (ver Figura 10).

De manera específica, se hizo uso del algoritmo Simple K-Means a partir de la librería weka, para definir 3 modelos basados en clustering. El primer modelo de clustering relaciona como atributos la estampa de tiempo y el valor del ritmo cardiaco, el segundo modelo relaciona como atributos la estampa de tiempo y el valor del nivel de oximetría, finalmente el tercer modelo relaciona como atributos la estampa de tiempo, el ritmo cardíaco y el nivel de oximetría. De acuerdo a lo anterior, en la Figura 10 se presentan los resultados de aplicar el primer modelo de clustering a la sesión de ejemplo de la Figura 9. Tal como se aprecia en la Figura 10, se encontraron dos centroides en dos iteraciones alrededor de los cuales se concentran los datos de la sesión de captura. Así, los centroides identificados por el algoritmo Simple K-Means fueron: (t1=14.4752, $\mathrm{hr} 1=77.1429)$ y ( $\mathrm{t} 2=43.9377$ y hr2=80.4839), mientras que el centroide general es ( $\mathrm{t}=29.9555, \mathrm{hr}=78.8983)$.

\section{Figura 10. Aplicación Modelo de Clustering}

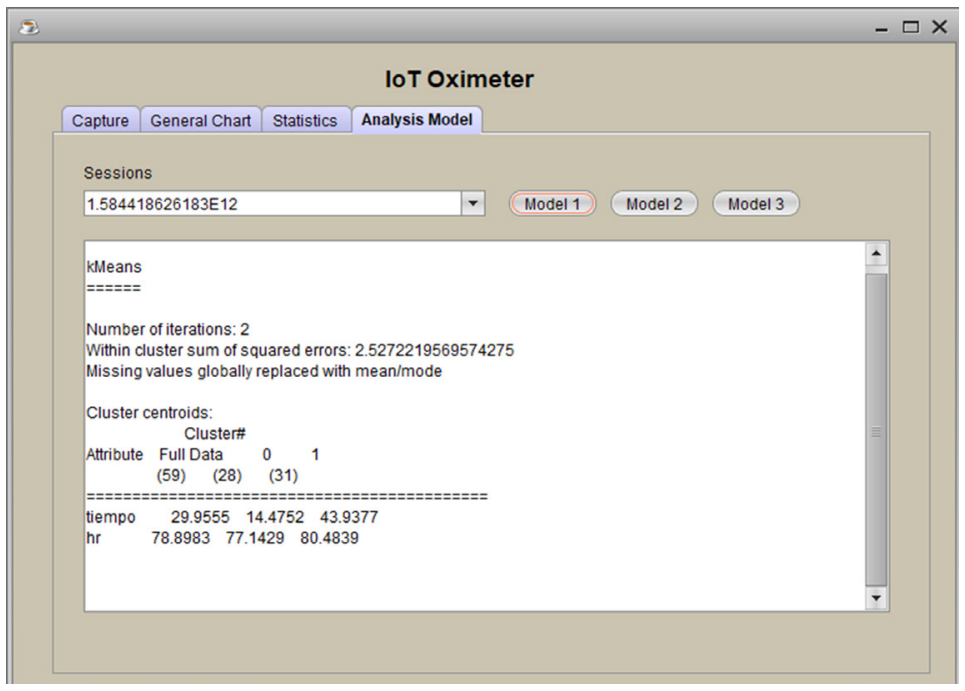

Fuente: Elaboración propia

En este orden de ideas, los resultados obtenidos de la validación de la arquitectura propuesta, permiten afirmar que sirve de referencia para el desarrollo de sistemas de monitorización y análisis de variables fisiológicas en el área de la asistencia médica, logrando aprovechar el uso de dispositivos comerciales IoT, al generar información de valor agregado, sin importar su naturaleza que los caracteriza por no ser abiertos, no proveer almacenamiento, ni incluir modelos de análisis de datos. 


\section{Conclusiones}

En este artículo se propuso como aporte una arquitectura IOT para la construcción de sistemas para la monitorización y análisis de variables fisiológicas en el servicio asistencial en salud. Esta arquitectura fue representada mediante tres vistas: de negocio, funcional y de implementación, cada una de las cuales incluye las cuatro capas de la arquitectura convencional de IOT (ver Figura 1). Los resultados obtenidos demuestran que esta arquitectura sirve de referencia para la construcción de sistemas para la captura, el monitoreo y el análisis de variables fisiológicas en el área de la salud.

La arquitectura plantea a nivel de la vista de implementación, un conjunto de técnicas y tecnologías que permiten aprovechar el potencial de los diferentes dispositivos comerciales que no cuentan con librerías de desarrollo (acceso no abierto), al momento de obtener y transformarlos datos de las variables fisiológicas en información útil para médicos y profesionales del servicio asistencial en salud. Así mismo, estas técnicas y tecnologías pueden ser desplegadas en diferentes plataformas, de tal modo que pueden ser ejecutadas en equipos convencionales empleados en hogares y centros de salud.

Como instancia de la arquitectura propuesta, se desarrolló un prototipo de sistema de monitorización loT del nivel de oxígeno, partiendo de un oxímetro comercial. El sistema propuesto permite la obtención y decodificación de la trama de datos, así como el seguimiento gráfico, el almacenamiento de los datos y el posterior análisis de los datos haciendo uso de modelos basados en clustering. Estos modelos permiten determinar los centroides de las sesiones de captura realizadas alrededor de los cuales se concentran los datos, teniendo en cuenta la relación entre los atributos de estampa de tiempo, ritmo cardíaco y nivel de oximetría.

La arquitectura propuesta y el prototipo implementado a partir de esta, aprovechan las ventajas provistas por las librerías y herramientas de analítica de datos tales como: weka y scikit learn, las cuales permiten la implementación de diferentes modelos de análisis como: reglas de asociación, árboles de decisión, clustering, etc. Estos modelos permiten que el personal especializado obtenga información relevante a partir de los datos convencionales obtenidos por dispositivos comerciales. Así, conviene identificar la relación entre las variables fisiológicas y el diagnóstico médico con el fin de hacer estos modelos más efectivos y útiles.

Como trabajo futuro derivado de la presente investigación, se pretende ampliar la arquitectura propuesta en cuanto a la monitorización simultanea de múltiples pacientes con diferentes dispositivos wearables articulados a su espacio corporal, con el fin de automatizar el proceso de seguimiento y brindar apoyo en el diagnóstico de pacientes en centros médicos.

\section{Referencias bibliográficas}

1. S. Naveen and S. Hegde, "Study of IOT: Understanding IOT Architecture, Applications, Issues and Challenges," Int. J. Adv. Netw. Appl., pp. 477-482, 2016, [Online]. Available: http://www.ijana.in/Special Issue/ S105.pdf.

2. M. Conti, A. Dehghantanha, K. Franke, and S. Watson, "Internet of Things security and forensics: Challenges and opportunities," Futur. Gener. Comput. Syst., vol. 78, pp. 544-546, 2018, doi: 10.1016/j. future.2017.07.060.

3. F. Firouzi, K. Chakrabarty, and S. Nassif, Intelligent Internet of Things: From Device to Fog and Cloud. Springer Nature Switzerland, 2020. 
Arquitectura IoT para el desarrollo de sistemas de monitorización y análisis de variables fisiológicas en el área de asistencia médica

4. M. Barrio, Internet de las Cosas, 1.a edició. Madrid: Reuss, 2018.

5. I. Bonilla-Fabela, A. Tavizon-Salazar, M. Morales-Escobar, T. Guajardo-Muñoz, and C. I. LainesAlamina, “lot, El Internet De Las Cosas Y La Innovación De Sus Aplicaciones," VInculaTégica EFAN, no. 1, pp. 2313-2340, 2016, [Online]. Available: http://www.web.facpya.uanl.mx/Vinculategica/Revistas/R2/2313-2340 - Iot, El Internet De Las Cosas Y La Innovacion De Sus Aplicaciones.pdf.

6. L. Da Xu, W. He, and S. Li, "Internet of things in industries: A survey," IEEE Trans. Ind. Informatics, vol. 10, no. 4, pp. 2233-2243, 2014, doi: 10.1109/TII.2014.2300753.

7. D. Delgado, D. Girón, G. Chanchí, and K. Márceles, “Arquitectura loT para la identificación de personas en entornos educativos," Rev. Ibérica Sist. e Tecnol. Informação, vol. E17, pp. 841-853, 2019, doi: 1646-9895.

8. E. Quiroga, J. Sergio, C. Wilmar, and G. Chanchí, “Propuesta de una Arquitectura para Agricultura de Precisión Soportada en IoT," Rev. Ibérica Sist. e Tecnol. Informação, vol. 24, pp. 39-56, 2017, doi: 10.17013/ risti.24.39.

9. A. Kulkarni and S. Sathe, "Healthcare applications of the Internet of Things: A Review," Int. J. Comput. Sci. Inf. Technol., vol. 5, no. 5, pp. 6229-6232, 2014, doi: 0975-9646.

10. P. Sanmartín, K. Ávila, C. Vilora, and D. Jabba, “Internet de las cosas y la salud centrada en el hogar," Salud Uninorte, vol. 32, no. 2, pp. 337-351, 2016, doi: http://dx.doi.org/10.14482/.

11. D. Delgado, D. Girón, G. Chanchí, K. Márceles, and S. Dionizio, "Sistema para la Detección y Seguimiento de Afecciones Cardíacas Soportado en SBC," Rev. Ibérica Sist. e Tecnol. Informação, vol. E17, pp. 717-728, 2019, doi: 1646-9895.

12. A. Mutlag, M. Ghani, N. Arunkumar, M. Mohammed, and O. Mohd, "Enabling technologies for fog computing in healthcare IoT systems," Futur. Gener. Comput. Syst., vol. 90, pp. 62-78, 2019, doi: 10.1016/j. future.2018.07.049.

13. H. Cervantes and R. Kazman, Designing Software Architectures: A Practical Approach. Boston: Addison-Wesley Professional, 2016.

14. G. Chanchí and J. Arciniegas, Arquitectura para el despliegue de servicios interactivo de TV móvil. Sistemas de recomendaciones y búsqueda semántica. Popayán: Universidad del Cauca, 2018.

15. G. Chanchí, M. Giraldo, and C. Campo, “Herramienta para el seguimiento del estrés mental en pruebas de usabilidad," Rev. Ibérica Sist. e Tecnol. Informação, vol. E17, pp. 678-689, 2019, doi: 1646-9895.

16. C. Gutierrez-Ardila, J. Cubillos-Calvachi, J. Piedrahita-Gonzalez, C. Montenegro-Marín, and P. Gaona-García, “Sistema IOT para el auto-diagnóstico de enfermedades del corazón usando la evaluación matemática de la dinámica cardiaca basada en la teoría de la probabilidad," Rev. Ibérica Sist. e Tecnol. Informação, vol. E17, pp. 1-10, 2019, doi: 1646-9895.

17. B. Farahani, F. Firouzi, V. Chang, M. Badaroglu, N. Constant, and K. Mancodiya, “Towards fog-driven IOT eHealth : Promises and challenges of IoT in medicine and healthcare," Futur. Gener. Comput. Syst., vol. 78, pp. 659-676, 2018, doi: 10.1016/j.future.2017.04.036.

18. N. Kumar, "IоT Architecture and System Design for Healthcare Systems," in International Conference On Smart Technology for Smart Nation, 2017, pp. 1118-1123, [Online]. Available: https://ieeexplore.ieee.org/ stamp/stamp.jsp?arnumber $=8358332$.

19. Y. Jianwei, L. Yang, and L. Shu, “eBPlatform : An IoT-based System for NCD Patients Homecare in China," in 2014 IEEE Global Communications Conference, 2014, pp. 2448-2453, [Online]. Available: https:// ieeexplore.ieee.org/document/7037175.

20. G. E. Chanchí, M. Sánchez, and W. Y. Campo, "Sistema software para el análisis del estrés mental en test de usuarios," Campus Virtuales, vol. 7, no. 2, pp. 105-114, 2018, [Online]. Available: www. revistacampusvirtuales.es. 